\title{
Hereditary Motor and Sensory Neuropathy with Hypercapnic Respiratory Failure and Recurrent Rhabdomyolysis: A Case Report and Literature Review
}

\author{
Noor Sameh Darwich ${ }^{1 *}$, Michael J. Pedoto ${ }^{2}$, and Ehsan Shabbir ${ }^{3}$ \\ ${ }^{1}$ Miami Valley Hospital, Dayton, $\mathrm{OH}, \mathrm{USA}$ \\ ${ }^{2}$ Department of Physical Medicine and rehabilitation, Miami Valley Hospital, Dayton, OH, USA \\ ${ }^{3}$ Department of Internal Medicine and Neurology, Wright State University, Miami Valley Hospital, Dayton, OH, USA
}

*Corresponding author: Noor Sameh Darwich, Miami Valley Hospital, Dayton, $\mathrm{OH}$,

USA, Email: nsdarwich@gmail.com

Received Date: May 03, 2021

Published Date: May 17, 2021

\begin{abstract}
Hereditary motor and sensory neuropathy (HMSN), formerly known as Charcot-Marie-Tooth (CMT), is the most common inherited neuromuscular disease. It is characterized by a heterogeneous inheritance pattern and variable age of onset of symptoms and disease severity. HMSN primarily affects long peripheral nerves, leading to distal weakness and muscle atrophy involving lower and upper extremities, culminating in various degrees of disabilities. Phrenic nerve involvement resulting in diaphragm dysfunction and respiratory failure has been described in advanced HMSN disease. We describe a 39-year-old woman with HMSN and recurrent episodes of rhabdomyolysis who presented with hypercapnic respiratory failure. Her symptoms were thought to be due to diaphragm paralysis. She required intubation and mechanical ventilation and was extubated successfully and transitioned to noninvasive positive pressure ventilation (NIPPV). She was discharged home after recovery with NIPPV at night in addition to physical and occupational therapy.
\end{abstract}

Keywords: Hereditary motor and sensory neuropathy; Charcot-Marie-Tooth; Rhabdomyolysis; Creatine kinase; diaphragm paralysis; phrenic nerve; Noninvasive positive pressure ventilation; Respiratory failure

Abbreviations: HMSN: Hereditary Motor and Sensory Neuropathy; CMT: Charcot-Marie-Tooth; CK: Creatine Kinase; NIPPV: Noninvasive Positive Pressure Ventilation; NCVS: Nerve Conduction Velocity Study; EMG: Electromyography

\section{Introduction}

Diaphragmatic dysfunction due to phrenic nerve involvement in patients with hereditary motor and sensory neuropathy (HMSN) (formerly known as Charcot-Marie-Tooth [CMT]) is uncommon in the early stages of the disease [1]. However, respiratory compromise due to diaphragmatic dysfunction caused by phrenic nerve involvement can be silent and insidious with minimal symptoms such as orthopnea [2]. In advanced HMSN disease with diaphragmatic paralysis, patients can present with hypercapnic respiratory failure requiring ventilatory support [3]. The prevalence of obstructive and central sleep apnea is higher in patients with HMSN [4]. Mild elevation in serum levels of creatine kinase (CK) may

be due to denervated muscle with increase permeability of muscle membranes to enzymes such as CK. Recurrentepisodes of significant increases in serum CK level more than five times the upper limit of normal in patients with HMSN suggest rhabdomyolysis [5]. This condition could be triggered by prolonged immobilization from advanced physical disability, overuse of proximal hypertrophic extremity muscles, or a concomitant underlying genetic mutation associated with rhabdomyolysis [6].

\section{Case Report}

A 39-year-old caucasian woman with a history of HMSN presented with shortness of breath, right upper quadrantabdominal 
pain, and back pain associated with having tea-colored urine along with generalized muscle aches. She had been diagnosed with HMSN at age 8 without genetic testing. Her brother had HMSN as well. Her past medical history was notable for periodic episodes of weakness associated with rhabdomyolysis, obstructive sleep apnea (using a continuous positive airway pressure device at night), hypertension, and occasional dysphagia to solid food for several years. The patient's respiratory complaints started five years prior when she presented with respiratory distress and elevated serum CK, transaminases, and dark urine consistent with rhabdomyolysis. She developed acute kidney injury managed with intravenous hydration and noninvasive positive pressure ventilation (bilevel positive airway pressure) was used during her hospitalization. The patient had similar previous presentations at least twice in the last few years before the current presentation that required hospitalization for respiratory failure and rhabdomyolysis. She had baseline twopillow orthopnea, which increased recently to four pillows. The patient attributed her current condition to overexerting herself in the previous few days at work as she works at a library and walks with bilateral ankle-foot orthotics without an assistive device. She was taking acetaminophen for pain as needed.

Her previous spirometry in upright position five years prior showed severe restrictive dysfunction with forced vital capacity (FVC) $1.45 \mathrm{~L}$ (38\% predicted), forced expiratory volume in 1 second (FEV1), 1.22 L (38\% predicted), and FEV1/FVC (84\% predicted) consistent with a restrictive pattern. The patient was unable to perform spirometry in the supine position due to respiratory distress. Before the current presentation, she required moderate assistance for grooming and total assistance for lowerbody dressing and bed/chair/wheelchair transfer, and maximum assistance for rolling and supine-to-sitting positioning.

On admission to the hospital, her blood pressure was 142/96
mmHg, temperature $97.1 \mathrm{~F}(36.2 \mathrm{C})$, pulse 114 per minute, respirations 24 per minute, and oxygen saturation $100 \%$ on $2 \mathrm{~L} / \mathrm{min}$ oxygen per nasal cannula. Her physical examination was notable for claw-hand appearance of all fingers in both hands with fingers held in the flexed position but could be passively extended almost to the neutral position. Her motor examination revealed normal tone in bilateral upper and lower extremities. Deep tendon reflexes were $1 / 4$ in bilateral upper and lower extremities with no ankle clonus and absent Babinski reflex with intact light touch sensation while her sensory examination during a prior admission had shown impaired temperature and vibration sensation in bilateral upper and lower extremities. At current presentation, she could not sit up on her own without rolling over to one side and had difficulty getting into bed with intermittent numbness of her fingertips and intermittent burning of the top of her thighs lasting for 15-30 minutes that resolved spontaneously. Her chest examination was notable for bilateral diminished breath sounds at the bases.

Laboratory test results from the current and previous hospitalizations are displayed in Table 1. Hepatitis serology was negative with normal serum acetaminophen level and negative COVID-19 PCR nasal swab.

Ultrasound of the abdomen was unremarkable, and a chest $\mathrm{X}$-ray revealed low lung volumes with elevated bilateral diaphragms. Computed tomography angiography of the chest revealed no signs of pulmonary embolism but was notable for bilateral lower lobes atelectasis. The patient was diagnosed with rhabdomyolysis and started on intravenous fluid therapy. On day 4 of hospitalization, the patient became increasingly lethargic, confused with questionable seizure-like activity in the extremities and acute respiratory distress. Arterial blood gases with comparisons to previous hospitalizations are displayed in Table 2 .

Table 1: Laboratory test results from the current and previous hospitalizations.

\begin{tabular}{|c|c|c|c|c|}
\hline Variable & Normal Reference Range & 5 Years Prior & 4 Years Prior & Current Hospitalization \\
\hline CK (U/L) & $0-200$ & 29128 & 61783 & $>22,000$ \\
\hline ALT (U/L) & $0-60$ & 496 & 479 & 452 \\
\hline AST (U/L) & $0-46$ & 810 & 721 & 1295 \\
\hline GGT (U/L) & $0-75$ & & & 31.4 \\
\hline Aldolase (U/L) & $\leq 8.1$ & Large & Large \\
\hline $\begin{array}{c}\text { Urinalysis } \\
\text { Blood }\end{array}$ & Negative & & $3+$ \\
\hline
\end{tabular}

Table 2: Arterial blood gases from current and previous hospitalizations.

\begin{tabular}{|c|c|c|c|c|}
\hline Variable & Normal Reference Range & 5 Years Prior & 4 Years Prior & Current Hospitalization \\
\hline Arterial blood gases & & $\mathrm{FiO}_{2} 30.0 \%$ & $\mathrm{FiO}_{2} 28.0 \%$ & $\mathrm{FiO}_{2} 100 \%$ \\
\hline $\mathrm{pH}$ & $7.35-7.45$ & 7.42 & 7.32 & 7.06 \\
\hline $\mathrm{pCO}_{2}(\mathrm{~mm} \mathrm{Hg})$ & $35.0-45.0$ & 36.2 & 51 & $>98.3$ \\
\hline $\mathrm{PO}_{2}(\mathrm{~mm} \mathrm{Hg})$ & $80.0-100.0$ & 83.1 & 112 & 242 \\
\hline $\mathrm{HCO}_{3}(\mathrm{mEq} / \mathrm{l})$ & $22.0-26.0$ & 23 & 25.7 & 36.2 \\
\hline
\end{tabular}


The patient required intubation and mechanical ventilation and was transferred to the intensive care unit. An electroencephalogram revealed moderate nonspecific cerebral dysfunction of the left frontotemporal and right mesial frontal regions with severe nonspecific encephalopathy but no seizure or epileptiform discharges. Computed tomography of the head was unremarkable, and echocardiography revealed normal systolic function with ejection fraction $63 \%$, grade 2 diastolic dysfunction with normal valve structure, and normal right atrial and ventricular function. She was weaned off the ventilator and was successfully extubated to noninvasive positive pressure ventilation (NIPPV) with a face mask. Her serum CK levels trended down during hospitalization. The patient had a history of occasional swallowing dysfunction with a solid diet and reported an event in which she nearly choked on solid food at a restaurant and had to undergo the Heimlich maneuver. Videofluoroscopy revealed laryngeal penetration with thin liquids without evidence of tracheal aspiration and moderate residue within the vallecula and piriform sinuses. The patient was started on a full liquid diet with aspiration precautions.

She was discharged home with oxygen at $4 \mathrm{~L} / \mathrm{min}$ during the day and NIPPV with average volume-assured pressure support (AVAPS) mode during sleep and the day as needed. The patient refused inpatient rehabilitation or any more testing, including rhabdomyolysis genetic testing, phrenic nerve electrophysiologic studies, ultrasound of the diaphragm, and the sniff test. She was referred to a genetics clinic for counseling. We believe that our patient with HMSN had developed hypercapnic respiratory failure due to diaphragmatic dysfunction caused by underlying phrenic nerve involvement. Her recurrent rhabdomyolysis was most likely due to prolonged immobilization from advanced physical disability combined with overuse of her denervated distal muscles and proximal hypertrophic muscles in the lower extremities. We cannot rule out an underlying coexisting genetic metabolic disorder causing episodic rhabdomyolysis.

\section{Discussion}

HMSN is the most common inherited disease affecting the peripheral nerves. It is characterized by a heterogeneous inheritance pattern and characteristic pathological, electrophysiological testing, clinical, and presenting features [7]. HMSN has a global prevalence and affects all races and ethnic groups. The prevalence of HMSN is $10-40$ per 100,000 , depending on geographic region. Although there is no ethnic predisposition, some rare HMSN subtypes are restricted to certain racial groups. HMSN may be less common among African Americans in the US [8,9]. The prevalence of HMSN might be underestimated because some patients are asymptomatic, and some mild cases go undiagnosed or are misdiagnosed [10].

HMSN can be inherited with an autosomal dominant, autosomal recessive, or X-linked pattern. However, new spontaneous mutations (de novo) have been reported [11,12]. At least 80 gene mutations have been identified that encode proteins involved in myelin production, function, integrity, and maintenance. Other genes mediate functions in Schwann cells, axons, and other components of peripheral nerves. Some mutations affect genes responsible for mitochondrial proteins, while some are associated with unknown gene mutations [13]. Mutation types associated with HMSN can be whole-gene duplications, deletions, or point mutations. The majority of cases are related to mutations in four genes, PMP22, MPZ, GJB1, and MFN2. Duplications of the PMP22 gene account for $70-80 \%$ of cases [14]. HMSN is classified as types 1 through 7 with many subtypes within each type. Types one and two are the most common types. CMT1A accounts for $55 \%$ of all cases and $66.8 \%$ of CMT1 cases [15], while CMT2A accounts for most CMT2 [16]. The underlying pathology in peripheral nerves involves demyelination, axonal degeneration, or both [17].

Age of onset of symptoms varies by subtype, usually in the first decade for CMT1 and in the second or third decades for CMT2. Early-onset CMT2 can occur. A late-onset form of CMT2 presents between 35 and 85 years of age, while X-linked HMSN can be present early in infancy [18]. Disease severity is highly variable, and some individuals may show only minimal signs of weakness while others are disabled. The most common initial presentation of HMSN is distal weakness and muscle atrophy of the lower and upper extremities manifesting as difficulty walking, with frequent falls, poor finger control, and difficulty in hand manipulation and writing. There are also gait problems such as high-stepped gait, toe and flat foot walking, difficulty running and keeping up with peers [19], and frequently sprained ankles. Distal muscle atrophy causes stork-leg deformities with foot drop and deformities such as pes cavus (high arched feet) or pes planus (flat foot). These findings may be associated with impaired sensation presenting later, and they tend to be less prominent. They include a gradual decrease of proprioceptive, touch, vibratory sensation, and temperature sensation in the foot and hand. These symptoms predispose the patients to skin breakdown, non-healing foot ulcers, bony deformities of the foot due to loss of protective sensation. In all four limbs, pain sensation usually remains intact, accompanied by diminished or absent deep tendon reflexes [20]. Muscle cramps, numbness, and burning sensation with pain in extremities develop later [21]. Postural tremors and gait ataxia have been described in infantile-onset HMSN. Later in the course, foot deformities such as hammer toes, claw hand, hand weakness, and atrophy of the intrinsic hand and foot muscles can develop. Proximal muscles are less affected, and hypertrophy can develop as a compensatory mechanism in the early stage. However, proximal muscle weakness can develop late in the course of advanced disease. Intellectual disability with mental retardation has been reported in CMTX2 and CMTX4 subtypes with infantile onset.

HMSN usually affects long peripheral nerves. However, cranial nerve involvement such as optic neuropathy, sensory neuronal hearing loss [22], and dysphagia have been reported in CMT4B1, CMTX4, and CMTX5. There is sometimes slow conduction of 
electrical and magnetic stimuli in the facial, accessory, and hypoglossal nerves without clinical impairment of the cranial nerves [23]. Patients with CMTX1 can experience episodic acute or subacute onset of central nervous system manifestations characterized by transient neurological deficits associated with white matter abnormalities on brain magnetic resonance imaging (MRI). These deficits include transient ischemic attack, hemiparesis, quadriparesis, dysarthria, leukoencephalopathy, and ataxia, which resolve spontaneously without permanent deficit.

Nevertheless, brain MRI changes may persist for several years after the event [24]. Oculomotor nerve involvement presenting with diplopia is rare. Vocal cord paralysis, which can be unilateral or bilateral and unrelated to the degree of muscular weakness in patients with HMSN [25], is due to recurrent laryngeal nerve involvement; this has been reported in CMT2C, CMT4A, and CMT6 [26], and presents as dysphonia or stridor [27], increasing the risk of aspiration. These findings can be the presenting complaint of HMSN, often diagnosed with laryngeal electromyographic studies consistent with peripheral neuropathy affecting the vagus nerve and its laryngeal branch [28,29]. Autonomic nervous system involvement in HMSN can manifest as autonomic disturbances such as bladder dysfunction and orthostatic hypotension [30,31].

Although respiratory muscle and diaphragmatic weakness are uncommon in early disease of HMSN [32], the phrenic nerve may be involved. Phrenic nerve involvement manifests as diaphragm dysfunction or paralysis presenting with orthopnea and hypoventilation with hypercapnia that worsens during sleep, causing headache, fatigue, difficulty concentrating, and daytime somnolence. These findings may be relatively common in advanced CMT1 and CMT2 subtypes, including CMT2C, CMT1A, CMT4A, and CMT4B1 [33,34]. Observational studies attributed diaphragmatic dysfunction in a patient with HMSN due to coexisting diabetes mellitus. However, phrenic nerve neuropathy with diaphragmatic dysfunction was later described in a patient with HMSN without diabetes mellitus [35]. Respiratory compromise due to diaphragmatic paralysis can be silent and insidious and should be suspected in patients with HMSN presenting with minimal symptoms of orthopnea. The diagnosis should be considered before impending respiratory failure occur [36].

Phrenic nerve electrophysiological study in patients with CMT1 shows slow conduction velocity in most patients, and $24-30 \%$ have abnormal spirometry findings with reduced FVC. Even fewer patients (8\%) reported having respiratory symptoms [37]. The leading underlying cause of respiratory failure in patients with HMSN is the denervation of the diaphragm and intercostal muscles [38]. However, thoracic cage abnormalities and neuropathic spinal arthropathy [39] with associated deformities such as scoliosis and kyphoscoliosis (reported in 42\% of children with HMSN) can contribute to the restrictive ventilatory defect.

Demyelination abnormalities of the phrenic nerve were observed in patients with HMSN with the axonal form [40].
Patients with HMSN and diaphragmatic dysfunction and chronic hypoventilation are at high risk for developing central sleep apnea with desaturation and hypercapnia, mostly during rapid eye movement sleep. The latter occurs due to deficits in the functional utility of the diaphragm in respiration during rapid eye movement (REM) sleep, while the activity of the intercostal muscles is markedly inhibited during this sleep stage [41]. Due to pharyngeal nerve neuropathy causing upper airway collapse, obstructive sleep apnea was also described in patients with HMSN with or without diaphragmatic dysfunction. There is an increased prevalence of obstructive and central sleep apnea in patients with HMSN. The severity of sleep apnea and underlying peripheral neuropathy in patients with HMSN are highly correlated. Restless leg syndrome and periodic leg movement disorder are found in many patients with CMT2 [42]. Diagnosis of HMSN depends on history, including detailed family history. Physical examination findings include distal muscle weakness/wasting accompanied by diminished deep tendon reflexes in upper and lower extremities with possible palpable enlargement of the peripheral nerve, which may occur secondary to nerve hypertrophy. There is also possible skeletal deformity, usually occurring at later stages of the disease. These findings are supported by electrophysiological studies, including nerve motor and sensory conduction velocity study (NCVS) and needle electromyography (EMG), followed by genetic testing to confirm the diagnosis [43]. Electrophysiological signs of demyelination can usually be found in all peripheral nerves, including those clinically unaffected. In the demyelinating form of HMSN (CMT1), NCVS findings vary depending on the phenotype. There is usually significant slowing of conduction velocity in both the motor and sensory nerves, mostly the motor nerves with values $<38-40 \mathrm{~m} / \mathrm{s}$ (normal range $>50 \mathrm{~m} / \mathrm{s}$ ). In the axonal-loss type (CMT2), the conduction velocity is normal or slightly reduced with markedly low compound muscle action potential amplitude (CMAP). Sensory nerve action potential (SNAP) amplitudes are usually reduced. NCVS may be reduced even in asymptomatic patients with HMSN. Classic findings on needle EMG indicate muscle denervation with the presence of fibrillation potentials. In general, there is no correlation between the severity of muscle weakness and motor impairment. The degree of slowing on nerve conduction studies can be detected even in asymptomatic individuals as early as one year of age. However, in some phenotypes like the CMTX, NCV slowing is proportionate to clinical severity [44].

Nerve biopsy was a critical diagnostic tool for diagnosing HMSN in the past. Currently, it is not routinely performed unless the clinical presentation is atypical when genetic testing does not reveal a molecular diagnosis and when NCVS and EMG are not diagnostic [45]. Classical findings of nerve biopsy in patients with HMSN show onion bulb appearance, which is a characteristic feature reflecting repetitive demyelination and remyelination in CMT1. In CMT2, sural nerve biopsy shows axonal degeneration and nerve fiber loss [46]. Muscle changes seen in HMSN are due to denervation and reinnervation with secondary myopathic changes, neurogenic 
atrophy, fatty infiltration, and edema but no inflammatory infiltrate. Muscle MRI reveals high signal intensity within skeletal muscles on T2-weighted images [47].

Diagnosis of diaphragmatic dysfunction due to phrenic nerve involvement in patients with HMSN is challenging [48]. Suggested studies include chest radiograph, spirometry, diaphragmatic ultrasound, chest fluoroscopy with the sniff test, and phrenic nerve electrophysiological studies such as cortical and posterior cervical magnetic stimulation [49]. Chest radiograph findings are neither specific nor sensitive but can show low lung volumes with the elevation of diaphragms. Vital capacity is a sensitive tool and reliable measure of respiratory function compared to supine and erect positions. FVC may fall $>25 \%$ from upright to supine, suggesting a significant compromise of diaphragmatic function in patients with neuromuscular diseases [50].

Other findings on pulmonary function testing in patients with HMSN include reduced maximal inspiratory pressure (MIP). This parameter reflects diaphragmatic strength. In HMSN, maximal inspiratory pressure is usually lower than $-60 \mathrm{~cm} \mathrm{H2O}$, and maximal expiratory pressure (MEP) can be mildly reduced. It should be noted that these tests are effort-dependent [51]. Fluoroscopy with sniff test involves quickly breathing in through the nose. It is a straightforward assessment of diaphragmatic motor function that confirms the absence of muscular contraction of the diaphragm during inspiration, where the diaphragm moves downwards as in healthy patients. There is a $1-2.5 \mathrm{~cm}$ excursion during normal quiet breathing, and in deep breathing, the excursion may be 3.6$9.2 \mathrm{~cm}$. In patients with diaphragmatic dysfunction, the affected hemidiaphragm does not move downwards during inspiration, or paradoxical motion can occur. Sniff fluoroscopy is helpful in the diagnosis of unilateral diaphragmatic paralysis. However, in bilateral diaphragmatic paralysis, the test can give the false appearance of caudad displacement of the diaphragm due to accessory muscle contracture in bilateral diaphragmatic paralysis [52]. Ultrasonography of the diaphragm with static measurement of the diaphragm thickness and changes in thickness during inspiration has been used to confirm diaphragmatic paralysis. However, the test is operator-dependent and has not been adopted for standard evaluation of diaphragm paralysis. Phrenic nerve enlargement sometimes is revealed on ultrasound in patients with HMSN [53].

Several electrophysiological studies are used to diagnose diaphragmatic paralysis in neuromuscular diseases with phrenic nerve neuropathy. Nevertheless, these diagnoses can be challenging, and expertise in interpretation is required [54]. Phrenic nerve stimulation testing accompanied by measurement of diaphragm CMAP using transcutaneous electrical stimulation or cervical magnetic stimulation of the phrenic nerve in the neck while recording diaphragm CMAP at the chest wall surface by two electrodes is usually an easy test with reliable results [55]. Another test is transdiaphragmatic pressure (Pdi), representing the difference between gastric pressure and pleural pressure. Pdi can be monitored using two trans-nasal thin-walled catheters. One is placed in the lower third of the esophagus above the diaphragm to assess changes in pleural pressure, and the second is placed in the stomach to measure abdominal or gastric pressure. Pdi can be measured during peak tidal volume inspiration and after phrenic nerve stimulation. At peak tidal volume inspiration, the value for Pdi is positive if the diaphragm is working normally and negative in diaphragmatic paralysis [56]. Diaphragmatic electromyography can also help differentiate between diaphragmatic paralysis due to underlying neuropathy and paralysis caused by myopathy. Nevertheless, it carries some risk from needle placement in the diaphragm. Dynamic MRI of the diaphragm is a promising noninvasive new technique for evaluating diaphragm excursion, motion, and volume [57].

Laboratory findings in HMSN may include elevated serum CK levels. As many as $50-75 \%$ of patients with motor neuron disorders such as amyotrophic lateral sclerosis (ALS) [58], spinalbulbar muscle atrophy (SBMA), and HMSN have elevated serum CK levels. These elevations are usually up to five times the upper limit of normal at rest. These values can be up to ten times the upper limit of the normal value after activity. These changes correspond to the degree of muscle denervation and compensatory reinnervation with muscle fiber hypertrophy and the degree of myopathic changes seen on muscle biopsy [59]. The significant elevation of CK in HMSN can be recurrent and episodic with flare-ups due to exacerbating factors such as period of prolonged immobility (which leads to rhabdomyolysis), emotional stress, alcohol use, pregnancy [60], and drugs such as vincristine, dapsone, metronidazole, and nitrofurantoin [61]. Vincristine, a chemotherapeutic agent used to treat various malignancies in both children and adults, can exacerbate and cause rapid deterioration of HMSN or reveal a previously undiagnosed case of HMSN [62]. In ALS, up to $40-75 \%$ of patients had elevated serum CK levels, while $80 \%$ of patients with SBMA has elevated serum CK levels. These elevations may be seen many years before the onset of clinical symptoms, while muscle biopsy shows changes of denervation atrophy without myopathic changes [63]. The precise underlying mechanisms of elevated CK levels in HMSN are not yet clear. However, one study suggested possible leakage of CK across cell membranes in denervated muscle tissue secondary to increased myocyte permeability or disturbed metabolism due to denervation and reinnervation processes. The latter lead to increased endogenous triphosphatase activity in the mitochondria of denervated muscle, leading to increased CK levels in patients with HMSN [64]. Finally, increased CK levels may result from the overuse of denervated muscle, increasing permeability, and compensated hypertrophic proximal muscle during ambulation in disabled patients with HMSN. The period of prolonged immobility may also contribute to the increased CK levels [65].

Correlations between elevated CK levels with the extent of muscle mass undergoing denervation and atrophy have been 
described. Prominent elevation of serum CK was more frequent among patients with the axonal phenotype of the MPZ mutation in HMSN [66]. In animal studies, muscles undergoing neurogenic atrophy were more permeable to $\mathrm{CK}$ than normal muscle. Between $3 \%$ and $30 \%$ of a subset of HMSN patients in a Japanese study were reported to have increased CK levels. Other muscle enzymes that may be elevated in HMSN are AST, ALT, lactate dehydrogenase, and aldolase [67]. Associated genetic mutations for rhabdomyolysis have been found in rare subtypes of HMSN. Recurrent episodes of rhabdomyolysis caused by LPIN1 gene mutations were described in a patient with CMT1A [68]. The C59T mutation in exon 1 of the MPZ gene has been associated with late-onset axonal type HMSN with elevated serum CK levels. Mitochondrial dysfunctions such as mitochondrial trifunctional protein (MTP) deficiency, a rare mutation autosomal recessive disorder of mitochondrial fatty acid in HADHB (hydroxyacyl-CoA dehydrogenase $\beta$-subunit) gene can cause recurrent rhabdomyolysis, and the presentation can mimic peripheral neuropathies such as HMSN [69,70,71]. Management of patients with HMSN includes genetic counseling and multispecialty supportive care to relieve symptoms, improve mobility, and improve flexibility and muscle strength to increase independence. There is currently no effective medical treatment to prevent or retard any of the pathophysiologies of HMSN, including abnormal myelin, myelin degeneration, and axonal degeneration [72,73].

Rehabilitation of HMSN patients relies on physical and occupational therapy to improve the symptoms and reduce or delay the risk of muscle contractures. These therapies involve moderate low-impact activities, including low-intensity exercises such as stretching, swimming, and weight training. These were found to be more beneficial than high-intensity exercise [74]. Orthoses to stabilize ankles, walking aids, fall precautions, ankle/leg braces, and podiatry consultations for appropriate footwear are needed occasionally. Various orthopedic procedures may be necessary to correct severe spinal or foot and joint deformities. These include osteotomy, arthrodesis, spinal surgery, and plantar fascia release, with pain management for musculoskeletal and neuropathic pain. Special care must be taken to avoid neurotoxic drugs such as neuromuscular blocking agents for patients undergoing anesthesia, and there must close monitoring postoperatively [75,76,77]. NIPPV is effective respiratory support when hypoventilation with hypercapnia and respiratory impairment occur in patients with HMSN. Long-term NIPPV is well tolerated in patients with neuromuscular disorders [78]. NIPPV can help manage muscle weakness with diaphragm dysfunction and sleep apnea [79]. NIPPV at night improved survival in patients with neuromuscular disease and respiratory failure. It also improved their quality of life. Continuous positive airway pressure therapy is not usually recommended in patients with neuromuscular disease, and bilevel positive airway pressure therapy is more effective than CPAP in these patients $[80,81]$. Diaphragm pacing has shown equivocal results in neuromuscular disease patients and has never been explicitly tested in HMSN patients.

Several potential experimental therapies for HMSN are being evaluated in clinical trials. These include the use of progesterone antagonists in animal models. These agents may slow disease progression [82]. High-dose ascorbic acid (vitamin C) [83] promotes myelination. However, it failed to show any significant benefit [84]. An ongoing trial of polytherapy (PXT3003) is studying the combination of baclofen, naltrexone, and sorbitol [85]. This combination was proposed to downregulate PMP22 overexpression. It may also improve myelination and axonal regeneration in patients with CMT1A, the most common form [86].

Stem cell and gene therapies are promising treatment options under investigation for patients with HMSN, including gene replacement, gene addition, suppression of mutant gene expression, or modulation of gene expression by using a highly modified virus as a vector to carry and insert a functional version of the gene to replace a defect gene into the cells such as Adenoassociated virus gene therapy technique. Implanting tonsil-derived mesenchymal stem cells (T-MSCs) after maturation into Schwann cells called T-MSC-SCs, then transplanting these cells into the muscles is another potential treatment for HMSN $[87,88]$. Although patients with HMSN should have a normal life expectancy, those with advanced disease complicated by respiratory failure have significantly shorter life expectancy than the normal population. Death in infancy in some subtypes of HMSN has been reported. Disabilities are common.

\section{Conclusion}

It is essential to have a high clinical suspicion of diaphragmatic dysfunction due to phrenic nerve involvement in patients with advanced HMSN disease. This complication should be recognized early in patients with orthopnea, daytime somnolence, and hypercapnia [89]. Closely monitoring of respiratory function status and sleep-disordered breathing is recommended in patients with HMSN [90]. Spirometry while supine and upright, phrenic nerve electrophysiological studies, the sniff test, and ultrasound of the diaphragm can be valuable diagnostic tools to aid in diagnosing diaphragmatic paralysis [91,92]. NIPPV has been used with success in patients with respiratory failure due to neuromuscular disorders in general and in HMSN disease in particular. It has been shown to reduce morbidity and mortality and improve quality of life $[93,94]$. While mild elevations in serum CK levels are expected in patients with HMSN, significant elevations should alert physicians to investigate underlying causes of rhabdomyolysis such as coexisting genetic metabolic disorders, medications or drugs, prolonged immobilization, overexertion, or infection [95].

\section{Acknowledgement}

None.

\section{Conflict of Interest}

No conflict of interest. 


\section{References}

1. Spiesshoefer J, Henke C, Kabitz HJ, Esra Akova-Oeztuerk, Bianca Draeger, et al. (2019) Phrenic nerve involvement and respiratory muscle weakness in patients with Charcot-Marie-Tooth disease 1A. J Peripheral Nerv Syst 24: 283-293.

2. Laroche CM, Carroll N, Moxham J, Stanley NN, Evans RJ, et al. (1988) Diaphragm weakness in Charcot-Marie-Tooth disease. Thorax 43: 478479.

3. Gilchrist D, Chan Ck, Deck JH (1989) Phrenic nerve involvement in Charcot-Marie-Tooth disease. Chest 96: 1197-1199.

4. Dematteis M, Pepin J-L, Jeanmart M, et al. (2001) Charcot-Marie-Tooth disease and sleep apnea syndrome: a family study. The Lancet 357: 267 272.

5. Mirzazadeh M, Maghsoodi N, Barron J (2014) Increased Serum Creatin Kinase. Clinical Chemistry 60(2): 301-306.

6. Welch KMA, Goldberg DM (1972) Serum creatine phosphokinase in motor neuron disease. Neurology 22:697-701.

7. McDonald CM (2012) Clinical Approach to the diagnostic evaluation of hereditary and acquired neuromuscular diseases. Phys Med Rehabil Clin N Am 23(3): 495-563.

8. Veerapaneni K. Charcot-Marie-Tooth Disease. Grand Rounds-2016 The University of Kansas Medical center, http://www.kumc.edu/ Documents/neurology/Charcot_Marie_Tooth_Disease.pdf

9. De Assis Aquino Gondim F, Lorenzo N (2019) Which patient groups have the highest prevalence of Charcot-Marie-Tooth (CMT) disease? Medscape.

10. Hattori N, Yamamoto M, Yoshihara T, Haruki Koike, Masanori Nakagawa, et al. (2003) Demyelinating and axonal features of Charcot-Marie-Tooth disease with mutations of myelin-related proteins (PMP22, MPZ and Cx32): a clinicopathological study of 205 Japanese patients. Brain 126 134-151.

11. Meggouh F, Bienfait HME, M A J Weterman, M de Visser, F Baas (2006) Charcot-Marie-Tooth disease due to a de novo mutation of the RAB7 gene. Neurology 67: 1476-1478.

12. Reilly MM, Hanna MG (2002) Genetic Neuromuscular Disease. J Neuro Neurosurg Psychiatry; 73(suppl III) ii12-ii21.

13. Kang PB (2021) Charcot-Marie-Tooth disease: Genetics, clinical features, and diagnosis. UpToDate: 1-54.

14. Barisic N, Claeys KG, M Sirotković-Skerlev, A Löfgren, E Nelis, et al. (2008) Charcot-Marie-Tooth Disease: A clinico-genetic Confrontation. Annals of Human Genetics 72: 416-441.

15. Shy ME, Balsamo J, J Lilien, J Kamholz (2001) A Molecular Basis for hereditary motor and Sensory Neuropathy Disorders. Current Neurology and Neuroscience Reports 1: 77-88.

16. Pipis M, Feely S ME, Polke JM, Mariola Skorupinska, Laura Perez, et al (2020) Natural history of Charcot-Marie-Tooth disease type 2A: a large international multicenter study. Brain 143: 3589-3602.

17. Reilly MM, Hanna MG (2002) Genetic neuromuscular Disease. J Neurol neurosurg Psychiatry 73(suppl II): ii12-ii21.

18. Bennett CL, Lawson VH, Brickell KL, K Isaacs, W Seltzer, et al. (2008) Late-onset hereditary axonal neuropathies. Neurology 71: 14-20.

19. Muglia M, Zappia M, Timmerman V, P Valentino, A L Gabriele, et al. (2001) Clinical and genetic study of a large Charcot-Marie-Tooth type 2A family from southern Italy. Neurology 56(1): 100-103.

20. Yoshimura A, Yuan JH, et al. (2019) Genetic profile and onset features of 1005 patients with Charcot-Marie-Tooth disease in japan. J Neurol Neurosurg Psychiatry 90: 195-202.

21. Klein CJ, Duan X, shy ME (2013) Inherited neuropathies: clinical overview and update. Muscle Nerve 48: 604-622.
22. Anzalone CL, Nuhanovic S, Amy P Olund, Matthew L Carlson (2018) Cochlear Implantation in Charcot-Marie-Tooth Disease: Case report and review of the Literature. Case Reports in Medicine 1-3.

23. Kumagai-Eto R, Kaseda Y, Shozo Tobimatsu, Takenori Uozumi, Sadatoshi Tsuji, et al. (2004) Subclinical cranial nerve involvement in hereditary motor and sensory neuropathy: a combined conduction study with electrical and magnetic stimulation. Clinical Neurophysiology 115: 1689-1696.

24. Wu N, said S, Sabat S, Matthew Wicklund, Mark C Stahl (2015) Recurrent Episodes of Stroke-like Symptoms in a patient with Charcot-Marie-Tooth Neuropathy X type 1. Case Rep Neurol 7: 247-252.

25. Holinger PC, Vuckovich DM, L D Holinger, P H Holinger (1979) Bilateral Abductor Vocal Cord paralysis in charcot-marie-Tooth Disease. Ann Otol 88: 205-209.

26. Lacy PD, Hartley BE, Rutter MJ, Cotton RT (2001) Familial Bilateral Vocal Cord Paralysis and Charcot-Marie-Tooth Disease type II-C. Arch Otolarygol Head Nech Surg 127: 322-325.

27. Jaiganesh T, Bentley A (2005) Hereditary motor and sensor neuropathy: a cause of acute stridor. Emerg Med J 22: 666-667.

28. Stojkovic T, Latour P, Viet G, Hurtevent JF, Antoon Vandenberghe, Vandenberghe A, et al. (2004) Vocal cord and diaphragm paralysis, as clinical features of a French family with autosomal recessive Charcotmarie-Tooth disease, associated with anew mutation in the GDAP1 gene. Neuromuscular Disorders 14: 261-264.

29. Dyck PJ, Litchy WJ, Minnerath S, T D Bird, P F Chance, et al. (1994) Hereditary Motor and Sensory neuropathy with Diaphragm and Vocal Cord paresis. Ann Neurol 35: 608-615.

30. Stojkovic T, Seze JD, O Dubourg, M.CArne-Bes, S Tardieu, et al. (2003) Autonomic and respiratory dysfunction in Charcot-Marie-Tooth disease due to Thr124Met mutation in the myelin protein zero gene. Clinical Neurophysiology 114: 1609-1614.

31. Nolano M, Manganelli F, Vincenzo Provitera MD, Chiara Pisciotta MD, Annamaria Stancanelli, et al. (2015) Small nerve fiber involvement in CMT1A. Neurology 84: 407-414.

32. Osanai S, Akiba Y, Nakano H, H Matsumoto, O Yahara, et al. (1992) Charcot-Marie-Tooth Disease with Diaphragmatic weakness. Internal Medicine 31(11): 1267-1270.

33. Snyder RW, Mishel HS, Christensen GC (1990) Phrenic nerve involvement in Charcot-Marie-Tooth disease. Chest 98: 1043.

34. Snyder RW (1990) Phrenic Nerve involvement in Charcot-Marie-Tooth disease. Chest 4: 1043.

35. Laroche CM, Carroll N, Moxham J, N N Stanley, R J Evans, et al. (1988) Diaphragm Weakness in Charcot-Marie-Tooth disease. Thorax 43: 478479.

36. Chan CK, Mohsenin V, Loke J, J Virgulto, M L Sipski, et al. (1987) Diaphragmatic Dysfunctiob in siblings with hereditary Motor and Sensory neuropathy (Charcot-Marie-Tooth Disease). Chest 91(4): 567570

37. Aboussouan LS, Lewis RA, Shy ME (2007) Disorders of Pulmonary function, sleep, and the upper Airway in Charcot-Marie-Tooth Disease. Lung 185: 1-7.

38. Nathanson BN, Yu DG, Chan CK (1989) Respiratory Muscle weakness in charcot-Marie-tooth Disease. Arch Intern Med 149: 1389-1391.

39. Anand N, Levine DB, S Burke, M Bansal (1997) Neuropathic spinal Arthropathy in Charcot-Marie-Tooth Disease. The Journal of Bone and Joint Surgery 7(8): 1235-1239.

40. Sagliocco L, Orlandi G, Calabrese R, Alberto Pellegrinetti, Ornella Baglini, et al. (2003) Electrodiagnostic Evidence of Phrenic Nerve Demyelination in Charcot-Marie-Tooth Disease 1 A. Am J Phys Med Rehabil 82(10): 754759. 
41. Teramoto S, Ishii T, matsuse T (2001) Central sleep apnoeas in patients with Charcot-Marie-Tooth disease. The Lancet 358: 70.

42. Dziewas R, Waldmann N, M Böntert, H Hor, T Müller, et al. (2008) Increased prevalence of obstructive sleep apnoea in patients with Charcot-Marie-Tooth disease: a case control study. J Neurol Neurosurg Psychiatry 79(7): 829-831.

43. Banchs I, Casasnovas C, Antonia Albertí, Laura De Jorge, Mónica Povedano, et al. (2009) Diagnosis of Charcot-Marie-Tooth Disease. Journal of Biomedicine and Biotechnology: 1-10.

44. Pareyson D, Scaioli V, Laura M (2006) Clinical and Electrophysiological Aspects of Charcot-Marie-Tooth Disease. NeuroMolecular Medicine 8: 3-22.

45. Prada V, massucco S, Venturi C, Alessandro Geroldi, Emilia Bellone, et al. (2019) Diagnostic value of sural nerve biopsy: retrospective analysis of clinical cases from 1981 to 2017. Frontiers in Neurology 1218: 1-8.

46. Vallat JM, Funalot B, Magy L (2011) Nerve Biopsy: requirements for diagnosis and clinical value. Acta neuropathol 121: 313-326.

47. Gallardo E, Garcia A, Onofre Combarros, José Berciano (2006) CharcotMarie-Tooth disease type IA duplication: Spectrum of clinical and magnetic resonance imaging features in leg and foot muscles. Brain 129 426-437.

48. Hogan GR (1988) Respiratory Muscle Dysfunction in hereditary Neuropathy. Arch Intern Med 148: 1707-1708.

49. Dube BP, Dres M (2016) Diaphragm dysfunction: diagnostic Approaches and Management Strategies. Journal of Clinical Medicine 5(113): 1-20.

50. White J, Bullock R.E, P. Hudgson, GJ Gibson (1992) Neuromuscular disease, respiratory failure and cor pulmonale. Postgrad med J 68: 820 823.

51. Mônica de Carvalho Alcântara, Marcello H Nogueira-Barbosa, Regina Maria França Fernandes, Geruza Alves da Silva, Charles Marques Lourenço, et al. (2015) Respiratory dysfunction in Charcot-Marie-Tooth disease type 1A. J Neurol 262: 1164-1171.

52. Celli BR (2021) Causes and diagnosis of bilateral diaphragmatic paralysis. uptoDate 1-19.

53. Marino-Ramirez MA. Ultrasound as a supplement to phrenic nerve conduction and needle electromyography (EMG) of the diaphragm. Neurology.

54. Similowski T, Straus C, V Attali, A Duguet, B Jourdain, et al. (1996) assessment of the Motoe Pathway to the Diaphragm Usig Cortical and Cervical magnetic Stimulation in the Decision-making process of Phrenic pacing. Chest 110(6): 1551-1557.

55. Similowski T, Mehiri S, A Duguet, V Attali, C Straus, et al. (1997) Comparison of magnetic and electrical phrenic nerve stimulation in assessment of phrenic nerve conduction time. J Appl Physiol 82(4): 1190-1199.

56. Delisa JA, Tipton NM (1979) Exercise effect on Creatine Phosphokinase elevation in Motor Neuron Disease. Arch Phys Med Rehabil 60: 397-400.

57. Sarwal A, Walker FO, Cartwright MS (2013) Neuromuscular Ultrasound for evaluation of the diaphragm. Muscle Nerve 47(3): 319-329.

58. Harrington TM, Cohen MD, J D Bartleson, W W Ginsburg (1983) Elevation of Creatine Kinase in Amyotrophic lateral sclerosis. Potential Confusion with Polymyositis. Arthritis and Rheumatism 26(2): 201-205.

59. Macia F, Le Masson G, Marie Rouanet-Larriviere, Desire Habonimana, Xavier Ferrer, et al. (2003) A prospective evaluation of phrenic nerve conduction in multifocal motor neuropathy and chronic inflammatory demyelinating polyneuropathy. Muscle \& Nerve 28: 319-323.

60. Pollock M, Nukada H, Kritchevsky M (1982) Exacerbation of CharcotMarie-tooth disease in pregnancy. Neurology 32(11): 1311-1314.

61. Weimer LH, Podwall D (2006) Medication-induced exacerbation of neuropathy in Charcot Marie Tooth disease. J Neurol Sci 242(1-2): 47 -
54.

62. Ibanez-Julia MJ, Berzero G, Reyes-Botero G, Maisonobe T, T Lenglet, et al. (2017) Antineoplastic agents exacerbating Charcot Marie Tooth disease:red flags to avoid permanent disability. ACTA Oncologia 57(3): 403-411.

63. Chahin N, Sorenson EJ (2009) Serum Creatine Kinase levels in spinobulbar muscular atrophy and Amyotrophic Lateral Sclerosis. Muscle \& Nerve 40: 126-129.

64. Carlson BM (2014) The Biology of long-term denervated skeletal muscle. Eur J Trans Myol-Basic Appl Myol 24(1): 5-11.

65. Welch KMA, Goldberg DM (1973) Response of serum Enzymes and other Biochemical Constituents to Strenuous Exercise in control Subjects and patients with Motor Neurone Disease. Journal of the Neurological Sciences 19: 225-234

66. Alexis Ascah, Jimmy Matas, Phillip F Gardiner, Eric Fontaine, Yan Burelle (2006) Muscle denervation promotes opening of the permeability transition pore and increases the expression of cyclophilin D. J Physiol 574(1): 319-327.

67. Yamamoto Y, Matsui N, Yu Hiramatsu, Yoshimichi Miyazaki, Hiroyuki Nodera, et al. (2017) Mitochondrial trifunctional protein deficiency: an adult patient with similar progress to Charcot-Marie-Tooth disease. Clinical Neurol 57(2): 82-87.

68. Nascimento A, Ortez C, Jimenez-Mallabrera C, Garcia-Villoria J, et al. (2011) Recurrent rhabdomyolysis caused by LPIN 1 gene mutation in a patient affected by Charcot-marie-Tooth 1A. Neuromuscular Disorders 21: 639-751.

69. Ceccanti M, Pozzilli V, Cambieri C, Laura Libonati, Emanuela Onesti, et al. (2020) Creatine Kinase and progression rate in Amyotrophic Lateral Sclerosis. Cells 9: 1174.

70. Vliet PV, Berden AE, Mojca KM van Schie, Jaap A Bakker, Christian Heringhaus, et al. (2017) Peripheral Neuropathy, Episodeic Rhabdomyolysis, and Hypoparathyroidism in a patient with Mitochondrial Trifunctional protein Deficiency. JIMD reports 37:101105.

71. Scalco RS, Gardiner AR, Robert Ds Pitceathly, Edmar Zanoteli, Jefferson Becker, et al. (2015) Rhabdomyolysis: a genetic perspective. Orphanet Journal of rare Disease 10: 51, 1-15.

72. McCorquodale D, Pucillo EM, Johnson NE (2016) Management of CharcotMarie-Tooth disease: improving long-term care with multidisciplinary approach. Journal of Multidisciplinary Healthcare 9: 7-19.

73. Iwata K, Scorrano L (2019) Finding a new balance to cure Charcot-MarieTooth 2A. The Journal of Clinical Investigation 129(4): 1533-1535

74. Dimitrova EN, Bozinovikj I, Ristovska S, Aleksandra Hadzieva Pejcikj, Aleksandra Kolevska, et al. (2016) The role of Rehabilitation in the Management of patients with Charcot-Marie-Tooth Disease: Report of two cases. Open Access Maced J Med Sci 4(3): 443-448.

75. Ohshita N, Oka S, Tsuji K, Hiroaki Yoshida, Shosuke Morita, et al. (2016) Anethetic management of a patient with Charcot-marie-Tooth Disease. Anesth Prog (63): 80-83.

76. Antognini JF (1992) Anaesthesia for Charcot-Marie-Tooth disease: a review of 86 cases. Can J Anaesth 39: 398-400.

77. Ortiz ER, Martinez E, Martin J, Maiza Lucas, Medina Jenny, et al. (2019) Spinal anesthesia in a patient with Charcot-Marie-Tooth disease undergoing orthopedic surgery: case report. Colombian journal of Anesthesiology 47(3): 180-183.

78. Mellies U, Ragette R, Schwake C, H Boehm, T Voit, et al. (2003) Long-term noninvasive ventilation in children and adolescent with neuromuscular disorders. Eur Respir J 22: 631-636.

79. Hill NS (1994) Noninvasive positive pressure ventilation in neuromuscular disease. Enough is enough! Chest 105: 337-338. 
80. Darquennes K, De Jonghe P, D Daems, W De Backer, J Verbraecken (2006) Intermittent positive airway pressure by nasal mask as a treatment for respiratory insufficiency in a patient wih charcot-Marie-Tooth disease. Acta Clin belg 61(4): 176-181.

81. Dias AS, Rinaldi T, Barbosa LG (2014) Noninvasive Ventilation with Pressure Support Ventilation as Strategy in Charcot-Marie-Tooth Disease: A Case Report. Am J Respir Crit Care Med 189: A6181.

82. Sereda MV, Meyer zu Horste G, Suter U, Naureen Uzma, Klaus-Armin Nave, et al. (2003) Therapeutic administration of progesterone antagonist in a model of Charcot-Marie-Tooth disease (CMT-1A). Nat Med Dec 9(12): 1533-1537

83. Passage E, Norreel JC, Noack-Fraissignes P, Véronique Sanguedolce, Josette Pizant, et al. (2004) Ascorbid acid treatment corrects the phenotype of a mouse model of Charcot-Marie-Tooth disease. Nat Med 10(4): 396-401.

84. Pareyson D, Schenone A, Fabrizi GM, Lucio Santoro, Luca Padua, et al (2006) A multicenter, randomized, double-blind, placebo-controlled trial of long-term ascorbic acid treatment in Charcot-Marie-Tooth disease type 1A (CMT-TRIAAL): the study protocol (EudraCT no.: 2006000032-27). Pharmacol Res 54(6): 436-441.

85. Chumakov I, Milet A, Cholet N, Gwenaël Primas, Aurélie Boucard, et al. (2014) Polytherapy with a combination of three repurposed drugs (PXT3003) down-regulates Pmp22 over-expression and improves myelination, axonal and functional parameters in models of CMT1A neuropathy. Orphanet Journal of Rare Diseases 9: 201.

86. Carvalho AAS, Vital A, Ferrer X, Philippe Latour, Alain Lagueny, et al. (2005) Charcot-Marie-Tooth disease type 1A, Clinicopathological correlation in 24 patients. Journal of the Peripheral Nervous System 10: 85-92.
87. Thenmozhi R, Lee J-S, Park NY, Byung-Ok Choi, Young Bin Hong, et al. (2020) Gene therapy options as new treatment for inherited peripheral neuropathy. Experimental Neurobiology 29(3): 177-188.

88. Rossor AM, Polke JM, Houlden H, Reilly MM (2013) Clinical implications of genetic advances in Charcot-Marie-Tooth disease. Nat Rev Neurol 9(10): 562-571.

89. Hardie R, Harding AE, Hirsch N, C Gelder, A D Macrae, et al. (1990) Diaphragmatic weakness in hereditary motor and sensory neuropathy. Journal of neurology, neurosurgery, and Psychiatry 53: 348-350.

90. Dyer EL, Callahan AS (1987) Charcot-Marie-Tooth Disease and respiratory Failure. Chest 92(5): 957.

91. Burakgazi AZ, Hoke A (2010) Respiratory muscle weakness in peripheral neuropathies. Journal of the Peripheral Nervous system 15(4): 307-313.

92. Santoro L, Manganelli F, Maio LD, F Barbieri, M Carella, et al. (2002) Charcot-marie-Tooth disease type 2C: a distinct genetic entity. Clinical and molecular characterization of the first European family. Neuromuscular disorders (12): 399-404.

93. Darquennes K, De Jonghe P, Daems D, De Backer W, Verbraecken J (2006) Intermittent Positive Airway Pressure by nasal mask as a treatment for Respiratory insufficiency in a patient with Charcot-Marie-tooth disease 61(4): 176-81.

94. Barbe F, Quera-Salva MA, J de Lattre, P Gajdos, A G Agustí (1996) Long-term Effects of Nasal Intermittent Positive-pressure Ventilation On Pulmponary Function and Sleep Architecture in patients with Neuromuscular Diseases. Chest 110(5): 1179-1183.

95. Zutt R, Van der Kooi AJ, Linthorst GE, R J A Wanders, M de Visser, et al. (2014) Rhabdomyolysis: review of the literature. Neuromuscul Diord 24(8): 651-659. 